\title{
GEOLOGÍA, CIENCIAS DE LA TIERRA, CIENCIAS DE LA NATURALEZA: PAISAJE DE UN APRENDIZAJE GLOBAL*
}

\author{
ANGUITA, F. \\ Departamento de Petrología y Geoquímica. \\ Facultad de Ciencias Geologicas. Universidad Complutense.
}

\begin{abstract}
SUMMARY
Notwithstanding its profound contributions to modern Science, Geology importance in Science Education has been (and still is) continuously dwindling. This situation is universal and has historic roots. At the same time, a reintegration of Geology with other sciences has been taking place, resulting in the Earth Sciences. The Earth Sciences concept is better adapted than Geology to new conceptual advances such as Systems Theory, Catastrophe Theory, or Chaos Physics. Nevertheless, caution is of essence when adapting those physico-mathematical theories to a Science whose methods are not mainly experimental but descriptive and historic. One of the present lines of advance in Earth Science Education is based on courses in which biologists and geologists together analyse Earth and Life systems. Viewing towards a farther horizon, we can find courses on Science integrating Biology, Chemistry, Geology and Physics concepts included in the new school curriculum. Although highly commendable in theory, the scheme is probably deemed to result in no real progress towards integrating Science, since it is not accompanied by deep changes in University curricula.
\end{abstract}

\section{INTRODUCCIÓN: LA CIENCIA INEXISTENTE}

«La ausencia de la geología entre las disciplinas cientificas es también un hecho que merece algún comentario. Aunque aparece ligada a la biología en aigunos paises, su presencia es realmente muy escasa. De hecho, son muy pocos los países que le conceden importancia de asignatura independiente.»

«Este proyecto de Formación del Profesorado en el área de Ciencias de la Naturaleza intenta cambiar todas las facetas de estas ciencias (biología, física, química...).»

«La respuesta del National Curriculum Council sería recomendar sólo tres líneas científicas, que serían biología, química y física, con los contenidos de Ciencias de la Tierra dividiéndose entre las tres.»

«El problema de la geología es interesante, ya que, mientras que en España se considera una asignatura científica, en gran número de países no se considera así, $y$ de hecho no hemos analizado los programas, ya que suele aparecer incorporada a la geografía.»

«Pobiación encuestada: profesores y estudiantes de física, 27; de química, 26, de biología, 41. Se incluye la respuesta de un profesor de geología.»
Las dos primeras de estas cinco citas corresponden a un proyecto de formación de profesores de ciencias en Latinoamérica a través de televisión (MEC 1993); la tercera, a un artículo de la revista de la Asociación de Profesores de Ciencias de la Tierra, británica (King 1993) la cuarta, a un estudio comparativo de los currícun los de ciencias en Iberoamérica (Nieda y Cañas 1992); y la quinta, a una encuesta realizada en el Institute of Education de Londres (Koulaidis y Ogborn 1989). Las cinco, por acción u omisión, describen la ausencia de la geología en el ámbito educativo, o incluso le niegan de hecho el carácter de ciencia.

Desde el punto de vista puramente científico, esta situación es sorprendente por varios motivos. Primero porque la geología sufrió hace tres décadas una espectacular revolución científica (el movilismo moderno), que no sólo la elevó al rango de ciencia predictiva, sino que hizo que sus contactos con la reina de las ciencias se estrechasen, llevando al primer plano del avance científico la especialidad de la geofísica. Segundo porque, en un mundo cada vez más preocupado por los problemas medioambientales, es difícil escapar de la realidad de que la mayor parte de los recursos que usa la civilización moderna, desde el acero al agua, se generan o se concentran 
a través de procesos geológicos; y porque, iguaimente, algunos de los más temibles riesgos naturales, desde terremotos a avalanchas, son procesos geológicos. Por último, y quizá lo más importante: la geología aporta a la cultura del hombre moderno el concepto de la antigüedad de la Tierra con homo sapiens como último invitado. Este anclaje en el tiempo tiene, como el anclaje espacial que suponen los modelos cosmológicos, un gran valor psicológico para que el hombre se sitúe en el universo: por ejemplo, como último llegado al planeta, no parece tener derecho a gastar todos los recursos acumulados en éste a lo largo de más de 4.500 millones de años. Que las escalas de tiempo usadas por geólogos $\left(10^{6}-10^{7}\right.$ años) e historiadores $\left(10^{2}-10^{3}\right.$ años) sean tan distintas no es en realidad un factor de separación, sino de conexión, porque los cambios globales ocasionados en los sistemas terrestres por la acción del hombre se ajustan también a la escala corta: para pasar de tratar la evolución de la litosfera o la biosfera a la evolución actual del medio ambiente y sus tendencias futuras basta con apretar un conmutador.

Si no hay una razón científica en la aparente tendencia de la geología a desaparecer, quizá este proceso tenga raíces históricas. Mayer (1991) argumenta que la situación de debilidad de esta materia en Estados Unidos se remonta a 1893, fecha en la que un comité de científicos notables (el Comité de los Diez) estableció las bases del primer currículo de ciencias en aquel pafs, integrado por cuatro materias: biologia, física, quimica... y geografía física, que luego fue suprimida. Uno se pregunta hasta qué punto la carencia geológica de los currículos latinoamericanos no es, al menos en parte, el reflejo de la misma situación en Estados Unidos.

En resumen, los grandes avances conceptuales en geolo* gía han llegado cuando esta ciencia estaba ya condenada a ser minoritaria. Estas situaciones tienden a acentuarse: siendo la geología la menos frecuentada de las especiaIidades científicas clásicas, Ios relativamente escasos profesores de geología se encuentran siempre en desven taja a la hora de defender su ciencia ante cualquier reforma curricular que casi siempre necesita sitio para las novedades científicas (en estos momentos, el medio ambiente, por ejemplo). En muchos casos, la poca geología presente se imparte con desgana por profesores de otras ramas. El círculo de este proceso que se autoalimenta se cierra con una cuota de alumnos cada vez más escasa.

El resto de este trabajo podría ser un lamento ante lo que la ciencia moderna está perdiendo al volver la espalda a la geología. En lugar de ello, sugeriré ideas para sacar a la geologia (y ello, en beneficio de toda la ciencia) del ghetto educativo en el que en este momento se halla confinada.

\section{GEOLOGÍA, GEOGRAFÍA Y NATURALEZA}

«...suele aparecer incorporada a la geografía». ¿Está justificada esta incorporación? No: geología y geografía no sólo no son sinónimos, sino que ni siquiera deberían solaparse. La geografía física se ocupa de los efectos de los procesos geológicos sobre el paisaje; la geología se dedica, utilizando el método analítico propio de la ciencia, a estudiar las causas de esos procesos, y de integrarlos en una comprensión global del planeta.

Los geólogos deberíamos reconocer, en cambio, que hasta hace poco tiempo la profesión geológica en su conjunto ha estado mediatizada por el punto de vista industrialista que requería de una ciencia que demostrase su utilidad económica a corto plazo: fue el auge de la geología prospectiva. Actualmente, con la mayor parte de los yacimientos minerales y de hidrocarburos ya descubiertos, y cuando la sociedad comienza a reparar más en la cara desagradable del crecimiento económico, el geólogo descubre que su profesión no es incompatible con el medio ambiente sino que la mejora de las interrelaciones hombre-medio requieren de su concurso, como sucede en el caso de los riesgos geológicos.

Probablemente ésta es una de las consecuencias del cambio que en las últimas décadas se ha producido en Occidente respecto a la naturaleza, cambio en el que la aspiración de control absoluto y explotación está cediendo poco a poco el paso al punto de vista oriental, cuya aspiración máxima es que el hombre viva en armonía con la naturaleza. Teniendo en cuenta que el mercado mundial de la protección del entorno mueve unos 24 billones de pesetas, es evidente que esta conversión masiva no puede ser totalmente idealista. Por ejemplo, las secciones de $I+D$ de Ias empresas con incidencia en temas medioambientales (o sea, casi todas) sc llaman ahora de medio ambiente e $\mathrm{I}+\mathrm{D}$; en muchos casos son, en realidad, oficinas de imagen y propaganda (Cortés 1993).

\section{UNA FUSIÓN RECIENTE: LAS CIENCIAS DE LA TIERRA}

En 1989, la Association of Teachers of Geology, británica, cambió su nombre por el de Earth Science Teachers' Association. ¿Qué había cambiado en la geología para justificar este tránsito? Para la propia asociación, los motivos del cambio eran tres:

- La moderna geología es sólo una de las especialidades científicas que nos sirven para comprender la Tierra. Junto a ella, la geofísica y la geoquímica han crecido hasta convertirse en ciencias separadas; y la meteorología, la climatología y la oceanografía (lo que los anglosajones suelen liamar conjuntamente ciencias de la Tierra fluida) son indispensables para comprender el funcionamiento de muchos procesos geológicos. Así pues, las ciencias de la Tierra son mucho más que geología.

- Este concepto ampliado es imprescindible para poner en contexto muchos de los problemas actuales relacionados con la Tierra, y específicamente los problemas medioambientales.

- En el aula de secundaria, una especialización muy estrecha (como la que se derivaría de la enseñanza de la 
geología clásica) podría disuadir a los alumnos de seguir una carrera científica.

En la I Conferencia Internacional sobre Educación y Formación en Geociencias, celebrada en Southampton, en abril de 1993, se planteó una reflexión paralela a las anteriores y según la cual en el período 1950-1975 la enseñanza en geociencias estuvo marcada por la influencia de las industrias extractivas de recursos geológicos (principalmente la petrolífera). Geotecnia y oceanografía fueron especialidades que surgieron al amparo de esta influencia. Sin embargo, desde 1975 el énfasis social se ha desplazado desde la explotación a la conservación (de los recursos y del medio); por ello, ahora están en alza especialidades de ciencias medioambientales y otras de frontera sin ninguna conexión industrial, como las ciencias planetarias; ambas líneas de avance son menos geológicas que las anteriores.

Existe otro aspectodel máximointerés que está parcialmente relacionado con este debate: ¿son idénticos Ios planteamientos didácticos que se pueden realizar en geología y en ciencias de la Tierra? La tradición de enseñanza experimental anglosajona en ciencias de la Tierra (que ha cristalizado, por ejemplo, en las «Science of the Earth 11-14' units» publicadas por la Asociación Británica de profesores de Ciencias de la Tierra) ha sido criticada por Hodson (1993) como rutinaria, mecanicista y de escasa incidencia en el proceso de aprendizaje. Sin embargo, vista desde un paf́s donde la experimentación (ingenua o sofísticada, integrada o no en el discurso de aula) ha sido un ave tan rara, la crítica puede parecer excesiva, teniendo en cuenta, especialmente, que un modelo experimental de una corriente de turbidez, pongamos por caso (Meléndez 1992), no exluye otros enfoques didácticos, como el histórico o la pequeña investigación. En este sentido, la propuesta de Mayer y Armstrong (1990) parece lo más sensata: para acceder al conocimiento deI sistema tierra, habría que usar de los tres métodos científicos: histórico, descriptivo y experimental. Como veremos enseguida, la informática podría ayudar de forma decisiva en esta sintesis.

En España se creó, en 1991, la Asociación Española para la Enseñanza de las Ciencias de la Tierra. Esta agrupa- ción heredaba un movimiento autónomo que, desde 1980, había estado organizando reuniones biaruales denominadas «simposios sobre enseñanza de la geología» Cuando en el último de ellos, celebrado en 1992, se propuso cambiar el nombre del siguiente por el de "Simposio sobre Enseñanza de las Ciencias de la Tierra», la respuesta fue de rechazo. Creo que es interesante preguntarse por qué. Sin đuda se requería más información, pero es probable que la mayoría de los profesores viese el cambio de denominación como una moda sin mayor contenido; e incluso así se sintiese insegura ante él. Para desmontar el argumento de que el cambio de denominación (de geología a ciencias de la tierra) sea oportunista, probablemente se pođría añađir, a los argumentos anteriores, otro, muy estrictamente científico: que las ciencias de la tierra admiten mucho mejor que la geología varios tipos de análisis cada vez más comunes en la ciencia moderna: la teoria de sistemas (Tabla 1), la teoría de catástrofes (Tabla II) y la fisica de los sistemas complejos ofísica del caos (Tabla III).

Aunque enunciados por separado, en la realidad, estas conexiones se solapan: podemos proponer como ejemplo la erosión de las playas debida a las tormentas invernales. Este fenómeno no es nuevo, pero sí lo es intentar entenderlo como el resultado de la superación de un umbral (es decir, una catástrofe en el sentido estricto de la palabra) de energía (la tormenta) en un sistema en cascada (energía solar, viento, oleaje, sedimentación litoral) en el que casi todos los elementos (el Sol, la atmósfera, la hidrosfera) son a su vez sistemas disipativos, estudiables no por la física lineal sino por la física del caos. Como podemos ver en las tablas II y III, muchos otros fenómenos que tienen Iugar en el sistema Tierra (incluyendo algunos tan aparentemente alejados de la física como son las extinciones masivas) podrían ser replanteados también con esta nueva luz.

Un último argumento, metodológico, a favor de las ciencias de la Tierra: la consideración de ésta como un sistema permite explotar con facilidad una visión holística del planeta. Visión que no es meramente ideal, ya que está apoyada materialmente por la plétora de datos que los satélites de recursos envían continuamente desde hace años. Estos datos son literalmente globales, y ello

Tabla I

Ciencias de la Tierra y teoría de sistemas.

* La Tierra como un sistema, integrado en el sistema solar, la Vía Láctea, el universo

* ... y compuesto por subsistemas: atmósfera, hidrosfera, biosfera, litosfera, astenosfera, núcleo...

* ... que evolucionan desde hace miles de millones de años, interaccionando entre sí en sus interfases (paisaje, suełos, costas)...

* ... produciendo campos (magnético) y gradientes (gravitacional, barométrico...) que dan lugar a procesos cuyas huellas podemos ver en rocas y fósiles.

En el apéndice se detalla una propuesta curricular mâs concreta, là «Earth Systems Education», basada en este concepto. 
Tabla II

Ciencias de la Tierra y teoría de catástrofes.

\begin{tabular}{|c|c|c|}
\hline Subsistemas & Ejemplos & Umbrales \\
\hline * Atmósfera & Huracanes & Superación de un gradiente térmico y bárico \\
\hline \multirow[t]{2}{*}{ * Hidrosfera } & Inundaciones & Superación de un gradiente térmico y bárico \\
\hline & T'sunamis & Alteración de la topografía submarina + morfología del litoral \\
\hline \multirow[t]{3}{*}{ * Litosfera } & Avalanchas & Ángulo de reposo, saturación \\
\hline & Megaseísmos & Litología, topografía, fluidos en el plano de falla \\
\hline & Erupciones paroxísmicas & $\begin{array}{l}\text { Viscosidad, volátiles del magma + campo } \\
\text { de esfuerzos en cánara magmática }\end{array}$ \\
\hline * En las interfases & \multicolumn{2}{|c|}{$\begin{array}{l}\text { Complejos: sistemas en cascada, por ejemplo } \\
\text { ¿Sobrepastoreo } \rightarrow \text { destrucción suelo } \rightarrow \text { aridez? }\end{array}$} \\
\hline
\end{tabular}

Tabla III

Ciencias de la Tierra y física de sistemas no lineales.

\begin{tabular}{|c|c|}
\hline Subsistemas & Sugerencias \\
\hline \multirow[t]{2}{*}{ * En el subsistema atmósfera } & $\begin{array}{l}\text { La predicción meteorológica como ejemplo de análisis } \\
\text { de un sistema cático a corto plazo. }\end{array}$ \\
\hline & $\begin{array}{l}\text { La predicción climática, ¿son las glaciaciones } \\
\text { atractores cáticos? }\end{array}$ \\
\hline * Kin el subsistema hidrosfera & $\begin{array}{l}\text { Las alteraciones en el sistema de corrientes marinas } \\
\text { como inestabitidades caóticas. }\end{array}$ \\
\hline * En el subsistema manto & $\begin{array}{l}\text { ¿Las «anomalias térmicas» en el manto terrestre } \\
\text { (y de otros planctas) como oscilaciones caóticas? }\end{array}$ \\
\hline * En el subsistema litosfera & $\begin{array}{l}\text { ¿Son los megaseísmos amplificaciones caóticas } \\
\text { de microseísmos? }\end{array}$ \\
\hline * En el subsistema biosfera & $\begin{array}{l}\text { ¿Son las extinciones oscilaciones caóticas de } \\
\text { un ecosistema sobrecargado? }\end{array}$ \\
\hline
\end{tabular}

no sólo en sentido geográfico sino también epistemológico, ya que la mayoría no son clasificables como datos físicos, químicos, biológicos o geológicos: son simplemente datos científicos sobre algún subsistema del sistema Tierra. Estos datos son resumidos y analizados por superordenadores, y luego pueden pasar en buena parte a disposición del sistema educativo median- te el uso de ordenadores con lectores de CD-ROMs, de forma que la siguiente revolución en tecnología educativa será la posibilidad de analizar en el aula conjuntos de datos sobre la evolución actual de subsistemas, siguiendo la metodología no experimental sino histórica (de escala corta, recordemos lo dicho en la introducción) típica de las ciencias de la Tierra. 
En resumen, las ciencias de la Tierra significan integrat en profundidad la geología con otras ciencias, y especialmente con la física; $y, a$ través de ello, obtener una mayor capacidad de comprender a fondo la Tierra en sus procesos. Un excelente ejemplo de la actualidad de este enfoque es la nueva materia «Ciencias de la Tierra y del Medio Ambiente» que se comenzará a impartir por vez primera durante el curso 1993-94 en el territorio MEC como materia propia deI nuevo bachillerato en su modalidad de ciencias de las naturaleza. Ésta ha sido la primera ocasión en que el Ministerio de Educación y Ciencia' ha implantado una materia que viola el acuerdo tácito de que cualquier ciencia experimental debe ser biología, o física, o química, o-más rara vez-geología.

Creo que esta anomalía no es del todo un fruto de la casualidad, ya que la Tierra es un lugar jógico de encuentro de la ciencia interdisciplinar: cuando nos acercamos lo bastante a las rocas, muchas de ellas resultan tener huellas de la evolución de la biosfera; a su vez, los minerales de estos fósiles se resuelven en redes cristalográficas, o sea en física y química. Esta conjunción nos lleva muy directamente al último aspecto.

\section{EL ESFUERZO FUTURO: LAS CIENCIAS DE LA NATURALEZA}

Ciencia coordinada, globalización, inter (o pluri, o multi) disciplinariedad, ciencia integrada... bellas expresiones que suelen encontrar entre el profesorado de ciencias de secundaria una acogida cautelosa, y esto por dos sencilios motivos: uno, que el profesorado tiene poca base científica externa a su titulación; y dos, que por tradición desconfía de que las autoridades educativas se vayan a empeñar en planes rigurosos de reciclado.

Por otra parte, las ideas sobre integración de ias ciencias en la enseñanza secundaria son tan persistentes como su atomización real en la superior. Esie movimiento de fusión floreció en los años 60 (patrocinado en parte por la UNESCO), pero los currículos actuales reflejan su fracaso. Se ha escrito que en aquel momento faltaba una idea nuclear, y se ha sugerido (Mayery Armstrong 1990) que el sistema Tierra podría cumplir este papel, con el argumento de que la mayoría de los procesos biológicos, físicos y químicos que el ciudadano del futuro deberá comprender para poseer una cultura científica se pueden aprender en el contexto de alguno de los subsistemas del sistema Tierra. Por ello, en mi opinión, los geólogos deberíamos convertirnos en los paladines más esforzados de la educación científica integrada.

En todo caso, la ciencia coordinada está ahí: en Ios contenidos del currículo oficial (MEC 1992) de la secundaria obligatoria, en la materia Ciencias de la Naturale$\mathrm{za}$, una materia que reúne contenidos de biología, de física, de geología y de química. Como la Naturaleza, por fin. Que debería ser impartida conjuntamente por los seminarios de ciencias naturales y de física y química. $Y$ que, en buena lógica científica, es indivisible. La mayoría de los 11 grandes bloques temáticos propuestos aún se dejan separar en biología (los seres vivos, Las personas y la salud), física (fuerzas, electricidad y magnetismo), geología (los materiales terrestres, cambios en el medio natural) y química (cambios químicos); pero otros son claramente mestizos, como: interacción entre los componentes abióticos y bióticos del medio natural, diversidad y unidad de estructura de la materia, o la tierra en el universo. Por último, el bloque denominado «Energía» se deja abordar desde cualquier ángulo. Algunos epígrafes de otros bloques (por ejemplo: el agua, desde el punto de vista del medio ambiente) comparten esta característica. Tampoco es casualidad que el aspecto medioambiental sea el más integrado: el medio ambiente es un patrimonio de todas las ciencias, y ninguna tiene derecho a intentar apropiárselo en exclusiva.

Creo que la idea de una materia científica coordinada es excelente; pero estoy mucho menos seguro de que sea compatible con la estructura actual de las titulaciones universitarias: los profesores de enseñanzas medias son biólogos, físicos, geólogos o químicos, y estas titulaciones abarcan alrededor de una cuarta parte de los contenidos de la nueva materia; y, aunque acceden a su condición de profesores superando un examen que comprende alrededor de la mitad de dichos conteridos, en general guardan lealtad a su especialidad, considerando, no sin razón, que ya han realizado un esfuerzo suficiente de ampliación de su base científica. Tanto más cuanto que han contemplado cómo, en la universidad, triunfa una furiosa microespecialización.

En algunos países se habla de una carrera global de ciencias para la formación de futuros profesores de ciencias en secundaria. Pero probablemente esta medida bienintencionada desde el punto de vista formativo diese lugar a una carrera "cenicienta» si los planes de estudio de tas carreras científicas clásicas no se modificasen en consonancia.

\section{CONCLUSIÓN: LA ENSEÑANZA DE LAS CIENCIAS DE LA TIERRA Y LAS CIENCIAS DE LA TIERRA}

En este punto, el lector tiene derecho a preguntarse si todo este revuelo estructural en el sistema educativo guarda alguna relación con la evolución reciente de la geología. Aparentemente, la respuesta es afirmativa: el término ciencias de la tierra se está extendiendo, en los títulos de las revistas científicas y de los departamentos o facultades de geología (en España, por ejemplo, la Facultad de Ciencias de la Tierra de la Universidad de Zaragoza). En la práctica, la sítuación es menos nítida: los geólogos de rocas duras (petrólogos, tectónicos) suelen interaccionar con los geofísicos y geoquímicos, pero bastante poco con los meteorólogos y climatólogos; mientras que los de rocas blandas (sedimentólogos, paleontólogos) colaboran con frecuencia con geoquímicos y oceanógrafos pero ven a los geofísicos como ajenos a su especialidad. En los nuevos planes de estudios de la geología universitaria en España, algunos (como el de la Universidad de Barcelona) tienen una fortísima carga de ciencias básicas, pero bastante menos 
otros (como el de la Autónoma de Barcelona, que sin embargo está muy orientado hacia las ciencias medioambientales). Materias que hacen referencia al sistema Tierra (como es, por ejemplo, la de Cambio Global) se han propuesto entre los nuevos cursos universitarios.

Más allá de las estructuras formales de la profesión, ¿hacia dónde van las ciencias de la Tierra? Podemos obtener una buena pista en el contenido de un reciente curso de verano de la Universidad Complutense (Universidad Complutense 1993), en el que se trató sobre impactos asteroidales y, muy en concreto, sobre el que, hace 65 millones de años, pudo extinguir las tres cuartas partes de las especies vivientes; también, sobre impactos climáticos, desde la perspectiva de la planetología comparada. El primer tema suscitó un debate tan enconado como sólo puede mostrar una ciencia viva, y es significativo que el máximo contraste surgiese entre la posición de los paleontólogos y la de los restantes especialistas (oceanógrafos, sedimentólogos y geoquímicos): un reflejo del contraste entre geología y ciencias de la Tierra, y un eco no muy lejano del largo debate sobre la continuidad o discontinuidad de la naturaleza. En cuanto al segundo tema, reunió a expertos en climatología terrestre con otros, más exóticos, en climas presentes y pasados de otros planetas terrestres. Unos y otros intentaron encontrar pautas para descifrar las tendencias del clima futuro de la Tierra y las interacciones de la civilización en este delicado sistema.

Los dos temas, y sus ramificaciones, representan con fidelidad lo que son hoy las modernas ciencias de la Tierra, en las que se distinguen dos líneas de avance: una en ciencia pura, que, basándose en el movilismo recién asentado, busca exportar este triunfante modelo al sistema solar más inmediato; y otra aplicada, que forma parte de las ciencias medioambientales y está basada en la triada de conceptos: recursos-riesgos-impactos.

\section{UN POCO DE LÓGICA, POR FAVOR}

El 31 de mayo de 1970, un terremoto agitó las escarpadas laderas de los Andes sobre Yungai, en Perú. Casi enseguida, el cerro comenzó a moverse en un alud de roca que se precipitó sobre la población: en sólo cinco minutos, más de 70.000 personas habían muerto. El proyecto de formación de profesores de ciencias en Latinoamérica no puede seguir ignorando las ciencias de la Tierra.

\begin{abstract}
NotA
'Mayer (1991) describe un intento similar realizado en Estados Unidos, aunque con alumnos de 15-16 años, a través de un curso denominado «Biological and Earth Science System», en el que los núcleos organizadores eran temas medioambientales.

* Ponencia presentada al IV Congreso internacional sobre investigación en la didáctica de las ciencias y de las matemáticas, celebrado en Barcelona en septiembre de 1993.
\end{abstract}

\section{APÉNDICE}

\section{Esquema básico del «Earth Systems Education» (Mayer 1991)}

Concepto 1. La Tierra es única, un planeta de rara belleza y gran valor.

Concepto 2. Las actividades humanas, colectivas e individuales, conscientes e inconscientes, están alterando gravemente el planeta Tierra.

Concepto 3. El desarrollo del pensamiento científico y de la tecnología incrementan nuestra capacidad para comprender y para utilizar la Tierra y el espacio.

Concepto 4. El sistema Tierra está compuesto de los siguientes subsistemas que interactúan entre sí: agua, tierra, hielo, aire y vida.

Concepto 5. El planeta Tierra tiene una edad de mâs de 4.000 miliones de años y sus subsistemas están evolucionando continuamente.

Concepto 6. La Tierra es un pequeño subsistema de un sistema planetario, dentro de un universo vasto y antiguo.

Concepto 7. Muchas personas siguen carreras relacionadas con el estudio del origen, procesos y evolución de Ia Tierra. 


\section{REFERENCIAS BIBLIOGRÁFICAS}

CORTÉS, H., 1993. Estudios ecológicos del entorno de las centrales térmicas de Endesa, Tecroambiente, 25, pp. 77-81.

HODSON, D., 1993. New thinking on the role of practical work in Science teaching, International Conference on Geoscience Education \& Training, Southampton, abstract volume.

KING, C., 1993. Fighting for Earth Science in the National Science Curriculum. A diary of ESTA's most recent battle, Teaching Earth Sciences, 18, pp. 56-59.

KOULAIDIS, Y. y OGBORN, J., 1989. Philosophy of Science: an empirical study of teachers' views, International Journal of Science Education, 11, pp. 173-184.

MEC, 1992. Secundaria Obligatoria. Ciencias de la Naturaleza. Madrid, $252 \mathrm{pp}$.

MEC, 1993. Proyecto de Formación Continua de profesores de Ciencias a través de la televisión educativa iberoamericana (borrador, 22 pp.).
MAYER, V.J., 1991. Earth Systems Education: origins and opportunities. The Ohio State University, pp. I-30.

MAYER, V.J. y ARMSTRONG, R.E., 1990. What every 17 year old should know about planet Earth: the report of a conference of educators and geoscientists, Science Education, 74, pp. 155-165.

MELENDEZ, I., 1992. Diferenciación química de un planta y formación de una corriente de turbidez: dos experiencias para presenciar los procesos geologicos en el laboratorio. III Congreso Geológico español y VIII Congreso Latinoamericano de Geologia. Salamanca, Actas, tomo I, pp. 439-446.

NIEDA, J. y CAN̉AS, A., 1992. Analisis comparado de los curriculos de Biología, Física y Química en Iberoamérica. Nivel medio. (Mare Nostrum: Madrid).

UNIVERSIDAD COMPLUTENSE, 1993. Catástrofes en la historia del Sistema Solar: un debate abierto. Cursos de Verano, pp. 197-201. (El Escorial. Programa-agenda: Madrid). 